\title{
DNA Damage Repair - Investigating the Conformations of DNA Ligase and PCNA
}

\section{Aleksandr Sverzhinsky and John M. Pascal}

University of Montreal

DNA damage repair is a central pillar of genomic integrity and all DNA repair pathways include DNA Ligase carrying out a 3-step reaction. Damaged DNA is not only produced from exogenous sources but also arises from normal cellular activities like genome replication. During replication, the lagging strand is synthesized as Okazaki fragments that require DNA Ligase to function efficiently. The trimeric proliferating cell nuclear antigen (PCNA) orchestrates DNA Ligase with nucleases and polymerases through their PCNA interacting peptide (PIP) motif. The PIP motif of DNA Ligase I is found in its unstructured N-terminus. An N-terminal truncation led to the crystal structure of DNA Ligase I in complex with nicked DNA. However, questions remain on Ligase domain rearrangements throughout the three steps of ligation and on its interactions with PCNA.

The hyperthermophilic archaeon Sulfolobus solfataricus provides a convenient model system of the DNA repair machinery found in all three branches of life. ssLigase lacks the unstructured N-terminus of human DNA Ligase I. Using a purification strategy that exploits the thermostability of the archaeal proteins, we purified S. solfataricus heterotrimeric PCNA and Ligase. ssPCNA was shown to stimulate ligation and co-eluted with ssLigase over gel filtration chromatography. We were able to visualize the ssLigasessPCNA-DNA complex by negative-stain electron microscopy (EM), providing insights into PCNA interaction. Using cross-linking strategies, we are pursuing a high resolution structure of this complex by cryo-EM. In parallel, we have initiated co-crystallization of ssLigase with DNA as well as the ssLigasessPCNA-DNA complex. With these two approaches, we will combine the atomic precision of X-ray crystallography with the ensemble domain dynamics of cryo-EM to obtain a comprehensive understanding of the final step of DNA damage repair. 